\title{
Qualidade de vida em pacientes da atenção primária do Rio de Janeiro e São Paulo, Brasil: associações com eventos de vida produtores de estresse e saúde mental
}

\author{
Quality of life of primary care patients in Rio de Janeiro and São \\ Paulo, Brasil: associations with stressful life events and mental health
}

\author{
Flávia Batista Portugal ${ }^{1}$ \\ Mônica Rodrigues Campos ${ }^{1}$ \\ Daniel Almeida Gonçalves ${ }^{2}$ \\ Jair de Jesus Mari ${ }^{2}$ \\ Sandra Lúcia Correia Lima Fortes ${ }^{3}$
}

${ }^{1}$ Escola Nacional de Saúde Pública, Fiocruz. R. Leopoldo Bulhões 1480, Manguinhos. 21041-210 Rio de Janeiro RJ Brasil. flaviabportugal@gmail.com ${ }^{2}$ Departamento de Medicina, Universidade Federal de São Paulo. São Paulo SP Brasil.

${ }^{3}$ Faculdade de Ciências Médicas, Universidade do Estado do Rio de Janeiro. Rio de Janeiro RJ Brasil

\begin{abstract}
Quality of life (QoL) is a subjective construct, which can be negatively associated with factors such as mental disorders and stressful life events (SLEs). This article seeks to identify the association between socioeconomic and demographic variables, common mental disorders, symptoms suggestive of depression and anxiety, SLEs with QoL in patients attended in Primary Care (PC). It is a transversal study, conducted with 1,466 patients attended in PC centers in the cities of São Paulo and Rio de Janeiro in 2009 and 2010. Bivariate analysis was performed using the T-test and four multiple linear regressions for each $Q o L$ domain. The scores for the physical, psychological, social relations and environment domains were, respectively, 64.7; 64.2; 68.5 and 49.1. By means of multivariate analysis, associations of the physical domain were found with health problems and discrimination; of the psychological domain with discrimination; of social relations with financial/ structural problems; of external causes and health problems; and of the environment with financial/ structural problems, external causes and discrimination. Mental health variables, health problems and financial/structural problems were the factors negatively associated with QoL.
\end{abstract}

Key words Quality of life, Stressful life events, Common mental disorder, Anxiety and depression, Primary care
Resumo A qualidade de vida (QV) é um constructo subjetivo, que pode ser associado negativamente a fatores como os transtornos mentais e os eventos de vida produtores de estresse (EVPE). O objetivo deste artigo é identificar a associação entre variáveis demográficas, socioeconômicas, transtorno mental comum, sintomas sugestivos de ansiedade e depressão, EVPE com QV na Atenção Primária (AP). Estudo transversal realizado com 1.466 pacientes atendido na AP, nos munícipios de São Paulo e Rio de Janeiro, em 2009 e 2010. Realizou-se análise bivariada por meio do Teste- $t$ e regressão linear múltipla para cada domínio de $Q V$. Os escores de $Q V$ para os domínios físico, psicológico, relações sociais e meio ambiente foram, respectivamente de, 64,7, 64,2, 68,5 e 49,1. Pela análise multivariada foram encontradas associações do dominio físico com os problemas de saúde e discriminação, do psicológico com discriminação, das relações sociais com problemas financeiros/estruturais, causas externas e problemas de saúde, $e$ do meio ambiente com problemas financeiros/ estruturais, causas externas e discriminação. As variáveis de saúde mental, os problemas de saúde e os problemas/financeiros estruturais foram os que se associaram negativamente à $Q V$.

Palavras-chave Qualidade de vida, Eventos de vida produtores de estresse, Transtorno mental comum, Ansiedade e depressão, Atenção primária 


\section{Introdução}

O conceito de estresse tem sido amplamente utilizado na literatura, sendo constantemente relacionado a efeitos deletérios à saúde. Lipp ${ }^{1}$ define estresse como "uma reação psicológica, com componentes físicos, mentais e químicos, a determinados estímulos que irritam, amedrontam, excitam e/ou confundem a pessoa". Assim, estresse é entendido como o conjunto de respostas visando a adaptação a eventos estressores aos quais o organismo é exposto ${ }^{2}$.

Nesse contexto, observa-se um interesse nas pesquisas atuais ${ }^{3,4}$ sobre os denominados eventos de vida produtores de estresse (EVPE), os quais são considerados eventos estressantes presentes no decorrer da vida (como separações, mudanças de moradia, internações, roubo e outros), que, quando acontecem, podem potencializar o processo de adoecimento dos indivíduos ${ }^{5}$, tanto aumentando a chance de ocorrência, quanto agravando os quadros preexistentes.

Atualmente, encontra-se na literatura a associação destes eventos com algumas condições de saúde, especialmente aquelas relacionadas à saúde mental, que incluem distintos transtornos psiquiátricos, tais como transtorno obsessivo-compulsivo ${ }^{6}$ e transtornos do pânico ${ }^{7}$. Um subgrupo importante é formado pelas condições psiquiátricas denominadas na literatura como transtornos mentais comuns (TMC), que englobam não só os transtornos depressivos ansiosos e as somatizações, mas também o sofrimento emocional inespecífico encontrado nos estudos comunitários e na atenção primária ${ }^{8,9}$. Nesta área, pode-se destacar o estudo sobre os EVPE e TMC realizado na Universidade do Estado do Rio de Janeiro (Pró-Saúde), o qual demonstrou a associação positiva entre transtornos mentais comuns (TMC) e EVPE, tais como: "dificuldades financeiras" e "ter sido vítima de agressão física"10. Outro estudo que se destaca é aquele produzido em Pelotas-RS, no qual os autores observaram que o maior número de EVPE aumenta a probabilidade de ocorrência de $\mathrm{TMC}^{11}$.

Relata-se, ainda, a influência destes eventos na qualidade de vida $(\mathrm{QV})$ da população. Entretanto, ainda são escassos os estudos sobre essa temática específica, sendo tais eventos geralmente associados a condições clínicas e psiquiátricas, como, por exemplo, HIV/Aids ${ }^{12}$, transtorno do estresse pós-traumático ${ }^{13}$, dependência química ${ }^{14}$ e asma ${ }^{15}$. Estudo realizado no Canadá com pessoas diagnosticadas com esquizofrenia apontou, por meio da regressão linear, que aborrecimentos diários foram associados a menores escores de $\mathrm{QV}^{16}$.

A QV é um constructo amplamente utilizado na literatura, entendida como uma condição eminentemente humana, abrangendo a vida familiar, amorosa, social e ambiental. O termo engloba inúmeros significados e, consequentemente, uma gama de $\operatorname{conceitos}^{17}$. A Organização Mundial de Saúde desenvolveu um conceito transcultural que define a QV "como a percepção do indivíduo de sua posição na vida no contexto da cultura e sistema de valores nos quais ele vive e em relação aos seus objetivos, expectativas, padrões e preocupações" ${ }^{\prime 18}$. É um conceito amplo que abrange a complexidade do constructo, inter-relacionando aspectos físicos, psicológicos, nível de independência, relações sociais e crenças. Logo, este conceito reflete a natureza subjetiva do constructo que está enraizada no contexto cultural e social, ao considerar, também, outros fatores, tais como a experiência pessoal, o meio ambiente e a situação de saúde ${ }^{19}$.

Dessa forma, o presente artigo visou identificar a associação entre variáveis demográficas, socioeconômicas, transtorno mental comum, sintomas sugestivos de ansiedade e depressão, EVPE com QV na Atenção Primária (AP) de pacientes atendidos nesta no Rio de Janeiro e em São Paulo, em 2009 e 2010.

\section{Metodologia}

\section{Tipo de estudo}

O presente artigo é um desdobramento do projeto de intervenção Avaliação de um modelo de capacitação em saúde mental na atenção básica: cuidados integrais na prática do matriciamento, cujo objetivo foi avaliar o impacto das capacitações em saúde mental nas atividades assistenciais na atenção primária. Essas capacitações eram voltadas para a integração das equipes de saúde da família e saúde mental, por meio da implementação das atividades de matriciamento e de intervenções terapêuticas em saúde mental na prática assistencial da Estratégia de Saúde da Família (ESF) ${ }^{20}$.

Neste trabalho, são apresentados os dados de dois estudos transversais do projeto supracitado nos munícipios de São Paulo (SP) e Rio de Janeiro (RJ), em 2009 e 2010. 


\section{Amostra}

A amostra analisada é formada por pacientes entre 18 e 65 anos, exceto grávidas e aqueles com déficit cognitivo, atendidos por equipes da ESF (médicos e enfermeiros) indicadas pelas prefeituras municipais de São Paulo e do Rio de Janeiro, para participarem das capacitações, sendo que o maior número profissionais envolvidos correspondia a este último município. Os dados foram coletados na sala de espera das unidades, antes da consulta com o profissional, por uma equipe de pesquisadores previamente treinados para tal atividade. O cálculo amostral inicial, realizado para o estudo original citado anteriormente ${ }^{20}$, considerou como variáveis desfechos aquelas que analisavam melhora na detecção de TMC pelos profissionais. Para tanto, foram testados cerca de 30 pacientes por equipe, resultando em 1.466, em dois estudos de cortes transversais (pré e pós-período de capacitação dos profissionais), da atenção primária do Rio de Janeiro (909 pacientes) e de São Paulo (557 pacientes), sendo que nenhum dos que participaram do primeiro estudo foi incluído no segundo. Todos participaram voluntariamente da pesquisa, após assinatura do Termo de Consentimento Livre e Esclarecido. Outros artigos, além do estudo original já citado ${ }^{20}$, utilizaram esta amostra para estudo dos $\mathrm{TMC}^{21,22}$ na atenção primária e sua associação com variáveis socioeconômicas e QV ${ }^{23}$.

\section{Instrumentos}

\section{Questionário socioeconômico e demográfico}

As variáveis socioeconômicas e demográficas foram obtidas por meio de um questionário fechado, utilizado previamente em outras duas pesquisas $^{10,24}$. Para a presente análise, optou-se pela dicotomização das variáveis, devido à homogeneidade da amostra, ou seja, em função do pequeno número de indivíduos em algumas categorias das variáveis. Foram selecionadas as seguintes variáveis para análise: sexo, faixa etária (até 40 anos e acima de 40 anos), grau de instrução (até a $4^{\circ}$ série e a partir da $4^{\text {a }}$ série do ensino fundamental) e renda familiar per capita (até 1 salário mínimo e acima de 1 salário mínimo).

World Health Organization Quality of Life Instrument, bref version (WHOQOL-bref)

O WHOQOL-bref é uma versão abreviada do World Health Organization Quality of Life Instrument (WHOQOL-100), contendo 26 questões referentes às duas últimas semanas antes da pesquisa. Esse instrumento de avaliação da qualidade de vida foi elaborado pelo grupo de trabalho liderado pela Organização Mundial de Saúde (WHOQOL group), responsável pelo seu desenho e pelas orientações para sua análise por meio da sintaxe proposta $^{18}$. A QV é analisada a partir dos domínios psicológico, físico, relações sociais e meio ambiente, sendo que em cada domínio o escore varia de 0 a 100 pontos. O domínio físico é composto por sete questões sobre presença de dor, fadiga, sono, dependência de medicação ou de tratamento, mobilidade, capacidade de trabalho e atividades da vida cotidiana. O domínio psicológico (com seis questões) engloba aspectos relacionados, por exemplo, autoestima, concentração e espiritualidade. O domínio relações sociais, por meio de três questões, aborda o suporte (apoio) social e a atividade sexual. O domínio meio ambiente (oito questões) investiga situações de segurança, ambiente no lar, recursos financeiros, cuidados de saúde, oportunidade de adquirir informações, recreação/lazer, ambiente físico (poluição/ruído/ trânsito/clima) e transporte. Há, ainda, duas perguntas referentes à avaliação individual da QV e outra sobre a satisfação com a própria saúde ${ }^{25}$. Ressalta-se que WHOQOL-bref possui propriedades psicométricas satisfatórias, sendo amplamente utilizado em pesquisas brasileiras ${ }^{25}$.

\section{Aferição de Transtornos Mentais Comuns (TMC)}

A detecção de TMC foi realizada por meio de dois instrumentos: um voltado para o rastreamento inespecífico de sofrimento emocional (o General Health Questionnaire - GHQ-12); e outro mais específico para sintomas depressivos e ansiosos (Hospital Anxiety and Depression Scale - HAD). Dessa forma, utilizaram-se quatro tipos de variáveis: duas para sintomas inespecíficos (com dois pontos de corte no GHQ); uma para casos sugestivos de ansiedade; e outra para casos sugestivos de depressão.

A seguir, são descritos os instrumentos utilizados.

1. General Health Questionnaire (GHQ-12)

O General Health Questionnaire (GHQ-12) foi desenvolvido por Goldberg and Blackwell ${ }^{26}$ e validado no Brasil a partir do Clinical Interview Schedule $^{27}$. Trata-se de um instrumento de rastreamento de transtornos mentais comuns (TMC), os quais são considerados "transtornos comumente encontrados nos espaços comunitários, cuja presença assinala uma alteração em relação ao funcionamento normal”28. O questionário é formado 
por 12 perguntas, cada qual com quatro opções de resposta, que se referem sempre ao período das duas últimas semanas antes da pesquisa ${ }^{29}$.

Como encontrado na literatura ${ }^{24}$, podem ser utilizados dois pontos de cortes para o rastreamento de TMC. Neste estudo, aqueles com 3 ou 4 pontos foram considerados positivos para transtorno mental comum e aqueles com 5 ou mais classificados como transtorno mental comum de intensidade grave (TMC-G).

2. Hospital Anxiety and Depression Scale (HAD)

A Hospital Anxiety and Depression Scale (HAD) foi desenvolvida para detecção de graus leves de transtornos afetivos (ansiedade e depressão), sendo validada e adaptada à realidade brasileira $^{30}$. São 14 questões referentes às últimas duas semanas antes da pesquisa, sendo sete sobre ansiedade e as outras sobre depressão, com respostas variando de 0 a 3 . Na análise, utilizaram-se os pontos de corte de $8 / 9$ para casos sugestivos de depressão e de ansiedade ${ }^{30}$.

\section{Eventos de Vida Produtores de Estresse (EVPE)}

O questionário para avaliar os EVPE é composto por 12 perguntas fechadas, com respostas dicotômicas, tendo como período de referência para ocorrência dos eventos os últimos 12 meses antes do preenchimento do questionário ${ }^{31}$.

São abordadas situações que podem interferir diretamente na saúde do indivíduo, tais como doença séria que resultou em afastamento das atividades habituais, internação hospitalar decorrente de doença ou acidente, morte de parente próximo, problemas financeiros severos, mudança forçada de moradia, separação/divórcio, agressão física e assalto/roubo. Outro ponto incluído refere-se aos aspectos relacionados à discriminação por cor ou raça, sexo, religião ou culto, opção ou preferência sexual, doença ou deficiência física, condição social ou econômica e idade ${ }^{10,31}$.

Para fins de análise, trabalhou-se inicialmente com os eventos individualizados, com exceção da discriminação, em que a presença de qualquer tipo foi a variável estudada. Em seguida, após constatar que esta população estava submetida a um alto número de EVPE (média de 1,6 EVPE por pessoa, sendo que $24,5 \%$ dos pesquisados relataram três ou mais), considerou-se mais adequada a análise por grupos aos quais as pessoas estavam sujeitas, visando estudar o efeito de diferentes tipos desses eventos. Assim, optou-se por organizar grupos de EVPE agregando aqueles com significados semelhantes. O primeiro grupo, Problemas Financeiros/Estruturais, agrega ques- tões referentes à dificuldade financeira e à mudança forçada de moradia. O segundo, Perda de Relação Afetiva, é composto por situações relativas ao rompimento de relação amorosa e à perda de parente próximo. O terceiro, Discriminação, engloba todas as questões supracitadas relacionadas a esse tema. O quarto, Problemas de Saúde, abarca os eventos de internação e outros relatos dentro do assunto. Por fim, o último grupo, Causas Externas, refere-se ao relato de agressão física, ferimento por arma de fogo ou branca, acidente de trânsito, presenciar ferimento a terceiros e catástrofe natural.

\section{Análise estatística}

Os dados foram analisados no programa Statistical Package for the Social Science - SPSS 17. Inicialmente, realizou-se uma análise bivariada por meio do Teste-t dos domínios de QV e as variáveis de socioeconômicas/demográficas, clínicas e EVPE, sendo aferidos seus p-valores. Posteriormente, as variáveis que foram estatisticamente significativas na análise bivariada, ao nível de significância de 10\%, foram incluídas em modelo de regressão linear múltipla. Foi desenhado um modelo para cada um dos quatro domínios de QV do WHOQOL-bref. As regressões foram realizadas mediante backward stepwise.

Os resultados dos quatro modelos finais da regressão linear são apresentados por meio do coeficiente de determinação (R2), dos coeficientes do modelo $(\beta)$ para cada variável de exposição e seu respectivo p-valor. Os resíduos de cada modelo foram analisados quanto às suas propriedades normais.

\section{Questões éticas}

O estudo de intervenção que deu origem a este artigo (Avaliação de um modelo de capacitação em saúde mental na atenção básica: cuidados integrais na prática do matriciamento) foi submetido ao Comitê de Ética e Pesquisa das Secretarias Municipais do Rio de Janeiro e de São Paulo, sendo sua realização considerada adequada à população humana. Além disso, o projeto que deu origem a este artigo foi submetido e aprovado pelo Comitê de Ética da Escola Nacional de Saúde Pública Sergio Arouca (Ensp/Fiocruz). Todos os participantes da pesquisa assinaram um Termo de Consentimento Livre e Esclarecido e receberam esclarecimentos sobre os objetivos da pesquisa, bem como a garantia de anonimato na divulgação dos resultados. 


\section{Resultados}

Foram pesquisados 1.466 indivíduos atendidos na atenção primária dos municípios de São Paulo e do Rio de Janeiro. Deste total, observou-se predominância de mulheres $(76,5 \%)$, da faixa etária acima de 40 anos (62,6\%), do grau de instrução a partir da $4^{a}$ série do ensino fundamental $(66,7 \%)$ e da renda familiar per capita inferior a um salário mínimo $(74,4 \%)$. Quanto às variáveis clínicas de saúde mental, os casos sugestivos de ansiedade foram encontrados em $37 \%$ dos pesquisados, casos positivos para TMC-G em 32\%, depressão em $25,1 \%$ e casos positivos de TMC em 20,5\%. No tocante à QV, foram encontrados escores médios de 64,7 para o domínio físico, 64,2 para o psicológico, 68,5 para o de relações sociais e 49,1 para o meio ambiente. Por meio da análise bivariada, encontraram-se menores escores de QV para as mulheres, para aqueles com renda familiar per capita inferior a um salário mínimo, para os casos positivos para TMC-G e para os casos sugestivos de ansiedade e depressão em todos os domínios do WHOQOL-bref (p-valor $<5 \%$ ). As pessoas acima de 40 anos apresentaram menores escores para os domínios físico e relações sociais, com diferença estatisticamente significante. Já entre aqueles com grau de instrução inferior à $4^{\mathrm{a}}$ série do ensino fundamental, observaram-se menores escores nos domínios físico, psicológico e meio ambiente ( $\mathrm{p}$-valor $<5 \%$ ) - Tabela 1 .

Sobre os EVPE, encontrou-se uma média 1,6 $( \pm 1,4)$ por indivíduo, sendo que apenas $26 \%$ dos pesquisados relataram nenhum evento no último ano. As maiores proporções de EVPE, individualmente, foram observadas para "dificuldades financeiras" (44,2\%), "falecimento de parente próximo" (22,6\%) e "problemas de saúde" (22,2\%). Quando analisados os grupos de EVPE, 47,1\% dos casos foram classificados em "Problemas Financeiros/Estruturais", 31\% em "Perda da Relação Afetiva", 27,9\% em "Discriminação", 27,1\% em "Problemas de Saúde" e 20, 9\% em "Causas Externas" - Tabela 2.

Tabela 1. Escores de Qualidade de Vida segundo características da população, RJ e SP, 2009/2010.

\begin{tabular}{|c|c|c|c|c|c|}
\hline \multirow{2}{*}{ Características } & \multirow[b]{2}{*}{$\%$} & \multicolumn{4}{|c|}{ Qualidade de vida } \\
\hline & & Físico & Psicológico & Relações sociais & Meio ambiente \\
\hline Escores médios de qualidade de vida & & 64,7 & 64,2 & 68,5 & 49,1 \\
\hline \multicolumn{6}{|l|}{ Sexo } \\
\hline Feminino & 76,5 & 63,7 & 62,9 & 67,9 & 48,2 \\
\hline Masculino & 23,5 & 67,9 & 68,8 & 70,3 & 52,2 \\
\hline \multicolumn{6}{|l|}{ Faixa Etária } \\
\hline Até 40 anos & 37,4 & 68,1 & $64,9^{*}$ & 70,2 & $48,8^{*}$ \\
\hline Acima de 40 anos & 62,6 & 62,7 & $63,9^{*}$ & 67,5 & $49,3^{*}$ \\
\hline \multicolumn{6}{|l|}{ Grau de Instrução } \\
\hline Até a $4^{\mathrm{a}}$ série & 33,3 & 61,6 & 61,5 & $68,3^{*}$ & 48,1 \\
\hline A partir da $4^{\mathrm{a}}$ série & 66,7 & 66,3 & 65,7 & $68,6^{*}$ & 49,6 \\
\hline \multicolumn{6}{|l|}{ Renda Familiar per capita } \\
\hline Até 1 Salário & 74,4 & 63,9 & 63,1 & 67,7 & 47,9 \\
\hline Acima de 1 Salário & 25,6 & 67,7 & 68,4 & 71,4 & 52,8 \\
\hline \multicolumn{6}{|l|}{ TMC } \\
\hline Não & 79,5 & $65,0^{*}$ & $64,2^{*}$ & $68,6^{*}$ & $49,3^{*}$ \\
\hline Sim & 20,5 & $63,6^{*}$ & $64,7^{*}$ & $68,1^{*}$ & $48,4^{*}$ \\
\hline \multicolumn{6}{|l|}{ TMC-G } \\
\hline Não & 68,0 & 70,4 & 70,0 & 72,5 & 52,3 \\
\hline Sim & 32,0 & 52,6 & 52,1 & 60,0 & 42,4 \\
\hline \multicolumn{6}{|l|}{ Ansiedade } \\
\hline Não & 63,0 & 70,3 & 70,2 & 72,5 & 52,5 \\
\hline Sim & 37,0 & 55,2 & 54,2 & 61,7 & 43,4 \\
\hline \multicolumn{6}{|l|}{ Depressão } \\
\hline Não & 74,9 & 68,7 & 69,1 & 71,2 & 51,8 \\
\hline Sim & 25,1 & 52,8 & 49,8 & 60,3 & 41,0 \\
\hline
\end{tabular}

"p-valor > 5\% para os casos em negrito e asterisco. 
Tabela 2. Escores de Qualidade de Vida segundo Eventos Produtores de Estresse. RJ e SP, 2009/2010

\begin{tabular}{|c|c|c|c|c|c|}
\hline \multirow{2}{*}{ EVPEs } & \multirow[b]{2}{*}{$\%$} & \multicolumn{4}{|c|}{ Qualidade de vida } \\
\hline & & Físico & Psicológico & Relações sociais & Meio ambiente \\
\hline \multicolumn{6}{|l|}{ Dificuldades financeiras } \\
\hline Sim & 44,2 & 60,4 & 61,0 & 64,0 & 45,1 \\
\hline Não & 55,8 & 68,1 & 66,9 & 72,0 & 52,3 \\
\hline \multicolumn{6}{|c|}{ Falecimento de parente próximo } \\
\hline Sim & 22,6 & 63,0 & $63,7^{*}$ & $68,9^{*}$ & $48,2^{*}$ \\
\hline Não & 77,4 & 65,2 & $64,5^{*}$ & $68,4^{*}$ & $49,4^{*}$ \\
\hline \multicolumn{6}{|l|}{ Problemas de saúde } \\
\hline $\operatorname{Sim}$ & 22,2 & 53,2 & 59,1 & 63,4 & 45,8 \\
\hline Não & 77,8 & 68,0 & 65,8 & 69,9 & 50,1 \\
\hline \multicolumn{6}{|l|}{ Internação } \\
\hline Sim & 11,7 & 54,0 & 59,5 & 64,0 & 46,4 \\
\hline Não & 88,3 & 66,1 & 64,9 & 69,1 & 49,5 \\
\hline \multicolumn{6}{|c|}{ Rompimento de relação amorosa } \\
\hline $\operatorname{Sim}$ & 11,6 & $63,0^{*}$ & $62,0^{*}$ & 64,7 & 47,1 \\
\hline Não & 88,4 & $64,9^{*}$ & $64,6^{*}$ & 69,0 & 49,4 \\
\hline \multicolumn{6}{|l|}{ Forçado a mudar de casa } \\
\hline $\operatorname{Sim}$ & 8,7 & 59,9 & 57,5 & 63,4 & 43,2 \\
\hline Não & 91,3 & 65,2 & 65,0 & 69,0 & 49,7 \\
\hline \multicolumn{6}{|c|}{ Presenciou alguém ser ferido } \\
\hline Sim & 7,5 & $62,2^{*}$ & $63,6^{*}$ & $66,2^{*}$ & 46,2 \\
\hline Não & 92,5 & $64,9^{*}$ & $64,4^{*}$ & $68,7^{*}$ & 49,3 \\
\hline \multicolumn{6}{|l|}{ Vítima de agressão física } \\
\hline $\operatorname{Sim}$ & 4,4 & 58,6 & 55,8 & 60,2 & 41,2 \\
\hline Não & 95,6 & 65,0 & 64,7 & 68,9 & 49,5 \\
\hline \multicolumn{6}{|c|}{ Vítima de assalto/vítima de violência } \\
\hline Sim & 5,1 & $64,2^{*}$ & $64,1^{*}$ & 63,5 & $47,1^{*}$ \\
\hline Não & 94,9 & $64,7^{*}$ & $64,3^{*}$ & 68,7 & $49,2^{*}$ \\
\hline \multicolumn{6}{|c|}{ Vítima de catástrofe natural } \\
\hline Sim & 3,3 & $60,2^{*}$ & 57,4 & $63,8^{*}$ & $45,8^{*}$ \\
\hline Não & 96,7 & $64,8^{*}$ & 64,5 & $68,6^{*}$ & $49,2^{*}$ \\
\hline \multicolumn{6}{|c|}{ Ferimento arma de fogo/branca } \\
\hline Sim & 0,6 & $63,1^{*}$ & $62,6^{*}$ & $56,6^{*}$ & $50,1^{*}$ \\
\hline Não & 99,4 & $64,7^{*}$ & $64,3^{*}$ & $68,5^{*}$ & $49.1^{*}$ \\
\hline \multicolumn{6}{|c|}{ Grupos de EVPEs } \\
\hline \multicolumn{6}{|c|}{ Problemas Financeiros/Estruturais } \\
\hline Sim & 47,1 & 60,9 & 61,1 & 64,3 & 45,4 \\
\hline Não & 52,9 & 68,1 & 67,1 & 72,2 & 52,5 \\
\hline \multicolumn{6}{|l|}{ Perda de Relação Afetiva } \\
\hline Sim & 31,0 & $63,5^{*}$ & $63,4^{*}$ & $67,9^{*}$ & 47,8 \\
\hline Não & 69,0 & $65,2^{*}$ & $64,7^{*}$ & $68,8^{*}$ & 49,7 \\
\hline \multicolumn{6}{|l|}{ Discriminação } \\
\hline Sim & 27,9 & 58,1 & 58,9 & 63,6 & 44,5 \\
\hline Não & 71,4 & 67,2 & 66,4 & 70,4 & 50,9 \\
\hline \multicolumn{6}{|l|}{ Problemas de Saúde } \\
\hline Sim & 27,1 & 53,5 & 59,7 & 63,7 & 46,0 \\
\hline Não & 72,9 & 68,9 & 66,0 & 70,3 & 50,3 \\
\hline \multicolumn{6}{|l|}{ Causas Externas } \\
\hline Sim & 20,9 & 61,8 & 61,5 & 64,4 & 45,6 \\
\hline Não & 79,1 & 65,4 & 64,9 & 69,5 & 49,9 \\
\hline
\end{tabular}

${ }^{*}$ p-valor $>5 \%$ para os casos em negrito e asterisco. 
Quanto à análise bivariada dos EVPE e QV, nota-se que a presença dos eventos associa-se a escores inferiores de QV nos pesquisados. Destaca-se que as questões referentes a "dificuldades financeiras", "problemas de saúde”, "internação", "forçado a mudar de casa" e "vítima de agressão física" apresentaram diferenças estatísticas em todos os domínios. Observando-se os grupos de EVPE, todos registraram diferenças estatísticas em todos os domínios, exceto o grupo "Perda de Relação Afetiva”, que só apresentou diferença no domínio meio ambiente (Tabela 3).

Por meio da análise multivariada, foi possível observar que as variáveis selecionadas para os modelos finais de cada domínio foram capazes de explicar a variabilidade $\left(\mathrm{R}^{2}\right)$ do domínio físico em 39\%, do psicológico em 43\%, das relações sociais em $16 \%$ e do meio ambiente em $23 \%$.

Vale destacar que todas as variáveis de exposição consideradas apresentaram associação negativa com $\mathrm{QV}$, com $\beta$ negativos, exceto grau de instrução e renda familiar per capita. As seguintes variáveis foram as mais expressivas para cada domínio: no físico - TMC-G $(\beta=-10,1$ e p-valor $=0,000)$, problemas de saúde $(\beta=-9,9$ e $p$-valor $=0,000)$ e discriminação $(\beta=-2,8$ e p-valor $=0,000)$; no psicológico - TMC-G $(\beta=-9,5$ e p-valor $=0,000)$, depressão $(\beta=-10,9$ e p-valor
$=0,000)$ e discriminação $(\beta=-1,9$ e $p$-valor $=$ $0,014)$; no relações sociais - TMC-G $(\beta=-6,5$ e $\mathrm{p}$-valor $=0,000)$, ansiedade $(\beta=-4,9$ e $\mathrm{p}$-valor $=$ $0,000)$, "Problemas Financeiros/Estruturais" $(\beta=$ $-4,1$ e p-valor $=0,000)$, "Causas Externas" $(\beta=$ $-2,8$ e p-valor $=0,015)$ e "Problemas de Saúde" $(\beta$ $=-2,0$ e p-valor =0,054); e no domínio meio ambiente - depressão $(\beta=-5,4$ e p-valor $=0,001)$, TMC-G $(\beta=-3,8$ e p-valor $=0,000)$, ansiedade $(\beta=-3,8$ e $p$-valor $=0,000)$. "Problemas Financeiros/Estruturais" ( $\beta=-3,5$ e $p$-valor $=0,000)$, "Causas Externas" $(\beta=-2,1$ e p-valor $=0,012)$ e discriminação $(\beta=-2,2$ e p-valor $=0,003)$.

\section{Discussão}

Os resultados do presente artigo mostram que as quatro dimensões de QV estão associadas a variáveis demográficas, socioeconômicas, clínicas e pelos EVPE. No entanto, o perfil de cada dimensão de QV é explicado só parcialmente pelas referidas vaiáveis. Sobre as variáveis socioeconômicas/demográficas, destaca-se a associação negativa da faixa etária mais elevada no domínio físico. Como já relatado na literatura ${ }^{31}$, os grupos etários mais velhos tendem a apresentar morbidades crônicas, por vezes cursando com diversas

Tabela 3. Modelos de regressão linear backward para os desfechos de QV em seus diferentes domínios (WHOQOL-bref). RJ e SP, 2009/2010.

\begin{tabular}{|c|c|c|c|c|c|c|c|c|}
\hline \multirow{2}{*}{ Variáveis independentes } & \multicolumn{2}{|c|}{ Físico } & \multicolumn{2}{|c|}{ Psicológico } & \multicolumn{2}{|c|}{ Relações sociais } & \multicolumn{2}{|c|}{ Meio ambiente } \\
\hline & $\beta$ & $\mathrm{p}$ & $\beta$ & $\mathrm{p}$ & $\beta$ & $\mathrm{p}$ & $\beta$ & $\mathrm{p}$ \\
\hline $\mathrm{R}^{2}$ & \multicolumn{2}{|c|}{0,39} & \multicolumn{2}{|c|}{0,43} & \multicolumn{2}{|c|}{0,16} & \multicolumn{2}{|c|}{0,23} \\
\hline Constante & 75,7 &, 000 & 66,1 &, 000 & 81,2 &, 000 & 52,7 &, 000 \\
\hline Idade & $-4,2$ & ,000 & - & - & $-2,9$ & 001 & - & - \\
\hline Grau de Instrução & 2,4 & ,004 & 3,2 &, 000 & - & - & 1,4 & ,039 \\
\hline Renda Familiar per capita & - & - & 2,0 &, 008 & & & 2,7 &, 000 \\
\hline TMC grave & $-10,1$ &, 000 & $-9,5$ &, 000 & $-6,5$ & ,000 & $-3,8$ &, 000 \\
\hline Ansiedade & $-5,1$ &, 000 & $-6,7$ &, 000 & $-4,9$ &, 000 & $-3,8$ &, 000 \\
\hline Depressão & $-6,3$ &, 000 & $-10,9$ &, 000 & $-3,8$ & ,002 & $-5,4$ &, 000 \\
\hline Problemas de saúde & $-9,9$ & ,000 & - & - & $-2,0$ & 054 & - & - \\
\hline Discriminação & $-2,8$ &, 001 & $-1,9$ &, 014 & - & - & $-2,2$ &, 003 \\
\hline Problemas Financeiros/Estruturais & - & - & - & - & $-4,1$ & 000 & $-3,5$ &, 000 \\
\hline Causas externas & - & - & - & - & $-2,8$ &, 015 & $-2,1$ &, 012 \\
\hline
\end{tabular}

${ }^{*} \mathrm{P}=\mathrm{P}$-valor do coeficiente Beta $(\alpha)$ no teste de Wald. $\beta$ é o coeficiente de cada variável independente por regressão. $\alpha$ é a constante em cada modelo segundo o domínio de QV investigado. As caselas em branco ( “-”) correspondem a variáveis não contempladas no modelo final. Variáveis adicionadas no modelo: Sexo $(0=$ Feminino/ $1=$ Masculino $)$, Idade $(0=$ Até 40 anos $/ 1=$ Acima de 40 anos), grau de instrução $\left(0=\right.$ Até $4^{\text {a }}$ série $/ 1=$ Acima da 4 a série do Ensino Fundamental $)$, Renda Familiar per capita $(0$ = Até 1 Salário-mínimo/ $1=$ Acima de 1 salário-mínimo $)$ TMC-G $(0=$ Não/ $1=\operatorname{Sim})$, Ansiedade $(0=$ Não/ $1=\operatorname{Sim})$, Depressão $(0$ $=$ Não/1 $=\operatorname{Sim})$, Discriminação $(0=$ Não/ $1=\operatorname{Sim})$, Problemas de Saúde $(0=$ Não/1 = Sim $)$, Perda de Relação Afetiva $(0=$ Não $/ 1=$ $\operatorname{Sim})$, Problemas Financeiros/Estruturais $(0=\mathrm{Não} / 1=\operatorname{Sim})$ e Causas externas $(0=\mathrm{Não} / 1=\operatorname{Sim})$. 
comorbidades, além de o próprio processo de envelhecimento gerar comprometimentos no bem -estar físico do indivíduo.

Existe a associação positiva do grau de instrução com os domínios físico, psicológico e meio ambiente e da renda familiar per capita com os psicológico e meio ambiente. Em estudo com usuários portadores de doenças crônicas de Unidades Básicas de Saúde de um município do Rio Grande do Sul, observou-se que aqueles classificados com classe econômica D e E apresentavam menores escores de QV ao serem comparados com a classe A. De acordo com os autores, as condições no Brasil, como baixo grau de escolaridade e distribuição de renda, contribuem negativamente para a QV da população ${ }^{32}$.

Quanto às variáveis de saúde mental, os resultados deste estudo corroboram aqueles encontrados na literatura ${ }^{33,34}$, os quais apontam que a presença de qualquer tipo de sofrimento associa-se a menores escores de QV. Destaca-se nos resultados do presente trabalho, um maior coeficiente de regressão linear (Beta) encontrado para TMC-G e os casos sugestivos de depressão em todos os domínios pesquisados, exceto no relações sociais. Tal fato mostra o impacto, na QV da população, de casos bem característicos de adoecimento psíquico, como a depressão. Berlim et al..$^{33}$ estudaram pacientes de um hospital universitário no sul do Brasil e observaram que a depressão foi o mais importante fator preditor para a redução dos escores de $\mathrm{QV}$ em todos os domínios pesquisados. Além disso, os autores reforçam que a gravidade do sintoma e o tratamento da depressão são fatores que podem refletir diretamente na QV. Outro estudo que relata essa associação foi realizado em 2009 e 2010, demonstrando que usuários de substâncias psicoativas (SPA) possuíam escores de QV inferiores quando comparados àqueles que não as usavam. Esse estudo ainda ressalta que os usuários de SPA eram quase duas vezes mais propensos a ter menores escores de $\mathrm{QV}$, independentemente de qualquer outra característica socioeconômica ${ }^{14}$.

A análise dos EVPE mostrou uma associação negativa do grupo "Problemas de Saúde" com os domínios físico e relações sociais. Há relatos na literatura de que a presença de alguma morbidade associa-se a baixos escores de QV, como, por exemplo, HIV/Aids ${ }^{12}$. Além disso, aponta-se que, conforme aumenta o número de morbidades, diminuem os escores de QV, especialmente nos componentes físico e mental ${ }^{35}$. A associação negativa encontrada com o domínio físico pode estar relacionada aos conceitos que embasam a construção deste, que é composto por situações referentes à dor física e à necessidade de atendimento médico ${ }^{25}$, questões que sofrem reflexos na presença de algum problema de saúde.

Para o domínio relações sociais, cabe destacar a importância do apoio social. Este é entendido como qualquer auxílio entre pessoas que se conhecem, o qual resulta em efeitos emocionais positivos, agindo como um relevante fator psicossocial e gerando maior satisfação com a vida $^{36}$. Dessa forma, sentir-se amado e cuidado está relacionado a níveis mais baixos de ansiedade, depressão e somatizações, bem como à melhor adaptação a situações de estresse ${ }^{37}$. Logo, o processo de adoecimento e a internação podem gerar comprometimento no convívio social, o que resultaria em menores escores no domínio relações sociais.

A discriminação associou-se negativamente a menores escores nos domínios físico e psicológico. Pesquisa realizada em Israel verificou a associação negativa entre discriminação e saúde física e mental (aferidos pelo Short Form Health Survey-12) entre não imigrantes judeus ${ }^{38}$. O estudo citado relaciona a QV com determinados grupos étnicos ${ }^{38}$, entretanto, há uma gama de situações que podem gerar discriminação, tais como gênero, orientação sexual, religião, aparência física, entre outras. O presente estudo não teve como foco analisar detalhadamente as causas de discriminação.

O grupo "Problemas Financeiros/Estruturais" associou-se negativamente à QV nos domínios relações sociais e meio ambiente. A literatura aponta que o domínio meio ambiente é o mais sensível a variações socioeconômicas ${ }^{39}$. Além disso, devese enfatizar que esse domínio abarca situações referentes a recursos financeiros ${ }^{25}$, que é uma das questões contempladas por esse grupo de EVPE. Por outro lado, a associação negativa desse grupo ao domínio relações sociais pode estar relacionada à mudança forçada de moradia, a qual afastaria $\mathrm{o}$ indivíduo do seu círculo de amizades.

Como era de se esperar, o grupo "Causas Externas" também associou-se negativamente à QV, especialmente nos domínios relações sociais e meio ambiente. Apesar desse grupo englobar desastres naturais, tais eventos foram pouco relatados pelos pesquisados, de tal forma que se torna, então, a violência um importante ponto de discussão, uma vez que esse grupo é mais representado pelas categorias "presenciar e/ou ser vítima de violência”. Há relato na literatura da associação da violência, de forma geral, a uma menor $\mathrm{QV}^{40}$. D'Souza et al. ${ }^{41}$ observaram que a presença de violência doméstica foi um fator pre- 
ditor que reduziu os escores de QV em todos os domínios aferidos pelo SF-36 (Short Form Health Survey-36).

"Perda de Relação Afetiva" foi o único grupo de EVPE que esteve ausente nos quatro modelos finais da regressão linear múltipla. Como não foi observada a associação deste evento diretamente na QV dos pesquisados, há a possibilidade de que o efeito deste possa estar incluso nas variáveis de saúde mental, como visto em estudo prévio realizado em Pelotas (RS), no qual o relato de separação conjugal e de morte de parente próximo associou-se à maior presença de distress ou mal -estar psicológico ${ }^{5}$, enquanto em outro a separação associou-se ao $\mathrm{TMC}^{10}$.

De modo geral, este estudo demonstrou que questões socioeconômicas, as variáveis de saúde mental e os eventos de vida produtores de estresse podem associar-se positiva ou negativamente à QV. Questões relacionadas à educação e renda necessitam de intervenções governamentais. Estas variáveis são denominadas determinantes sociais de saúde (DSS), que, segundo Carvalho e Buss $^{42}$, são fatores sociais, econômicos, culturais, étnicos/raciais, psicológicos e comportamentais que influenciam a ocorrência de problemas de saúde e seus fatores de risco na população. Assim, os resultados da presente pesquisa corroboram o sugerido pela promoção da saúde, já que os DSS, de nível macro, necessitam de políticas governamentais que visem à redução da inequidade social, a fim de gerar melhores condições de saúde.

Por outro lado, a associação negativa do transtorno mental na QV sugere que ações no próprio serviço de saúde podem gerar significativa melhora na vida do indivíduo. A assistência em saúde mental ainda é um desafio no Brasil. A QV permite identificar e priorizar problemas, uma vez que a sua mensuração oferece uma gama de informações sobre a saúde do indivíduo, possibilitando identificar em qual campo (ou domínio, no caso do WHOQOL-bref) deve-se atuar. Ademais, os resultados obtidos pela mensuração da QV permitem o compartilhamento de decisões entre profissional de saúde e paciente, a fim de identificar prioridades para o tratamento e facilitar o estabelecimento de metas realistas ${ }^{43}$. Dessa forma, a QV pode tornar-se um importante constructo na avaliação da assistência em saúde mental. Segundo Fortes et al. ${ }^{9}$, é necessário o treinamento dos profissionais da atenção primária para o manejo do transtorno mental. Além do diagnóstico e tratamento medicamentoso, o treinamento deve abordar habilidades adicionais, tais como estratégias de coping e intervenções psicossociais.

O conhecimento da influência de eventos estressores na população atendida pela atenção primária pode direcionar a assistência ao enfrentamento destas situações. A atenção primária é considerada a porta de entrada do sistema de saúde brasileiro, tornando-se responsável por prestar assistência integral e realizar atividades de educação e promoção da saúde ${ }^{44}$. Logo, ao atender estes indivíduos, a assistência deve-se direcionar aos impactos psicossociais que tais eventos podem gerar. Ressalta-se, também, que o sistema de saúde deve trabalhar conjuntamente com outros sistemas, como, por exemplo, o Sistema Único de Assistência Social (SUAS), que organiza ações de assistência social, visando a prevenção de riscos sociais e pessoais e a proteção daqueles já em risco ${ }^{45}$. Assim, a identificação de EVPE, como a violência, demanda esforços tanto do serviço de saúde como da assistência social.

Por fim, como ponto forte deste estudo, destaca-se o caráter multicêntrico em um estudo analisando o efeito de EVPE em população de baixa renda na atenção primária, no Brasil.

Destaca-se, como limitação, o uso de instrumentos apenas de rastreamento de transtornos mentais (GHQ e HAD), que detectam a possível existência de sintomas psiquiátricos e não seu efetivo diagnóstico. Assim, há a possibilidade de se detectarem falso-positivos, aumentando, desse modo, a proporção de casos de transtorno mental.

Além disso, pelo caráter seccional deste estudo, a exposição e o desfecho são aferidos simultaneamente, havendo a possibilidade de ocorrência de causalidade reversa. Por esse motivo, no presente artigo, não se pode pensar em associações causais tipo risco, limitando-se a discutir a associação existente entre os fatores estudados. As associações encontradas entre os diversos EVPE, TMC com QV indicam a necessidade de realização de estudos longitudinais, por meio dos quais será possível melhor identificar a influência destes fatores na vida da população no decorrer do tempo, de modo a elucidar a questão da causalidade reversa.

Ademais, as variáveis escolhidas explicaram parcialmente o desfecho, sendo necessária a investigação de um modelo teórico mais amplo, incluindo outras, como, por exemplo, o acesso ao serviço de saúde, suas características e a satisfação do usuário. 


\section{Considerações finais}

Apesar das limitações, o presente estudo apontou que algumas variáveis associaram-se significativamente à QV da população atendida pela atenção primária, sendo o transtorno mental comum, os casos sugestivos de ansiedade e de depressão, os problemas de saúde e os financeiros/estruturais as variáveis que mais impactaram no desfecho.

Devido à alta prevalência e à sua associação com a QV, torna-se importante que os profissio- nais de saúde sejam capacitados para identificar e tratar estes transtornos na AP, minimizando seu impacto na QV. Além disso, alguns pontos requerem atuações governamentais que visem à redução da inequidade social. Observou-se que os problemas financeiros/estruturais associaram-se a menores escores de QV, sugerindo, assim, que políticas voltadas para uma melhor distribuição de renda possam gerar efeitos benéficos à saúde da população, em especial na QV.

\section{Colaboradores}

FB Portugal participou da análise dos dados e interpretação dos resultados, redação, revisão e aprovação final do artigo; MR Campos da concepção do projeto, análise dos dados e interpretação dos resultados, redação, revisão e aprovação final do artigo; DA Gonçalves da concepção do projeto, coordenação do campo de pesquisa, análise dos dados e interpretação dos resultados, revisão e aprovação final do artigo; JJ Mari da concepção do projeto, revisão e aprovação final do artigo; SLCL Fortes da concepção do projeto, coordenação do campo de pesquisa, análise dos dados e interpretação dos resultados, redação, revisão e aprovação final do artigo. 


\section{Referências}

1. Lipp M. Stress e suas implicações. Estudos de Psicologia 1984; 1(3):5-19.

2. Glina DMR. Modelos teóricos de estresse e estresse no trabalho e repercusões na saúde do trabalhador. In: Glina DMR, Rocha LE, editor. Saúde mental no trabalho: da teoria à prática. São Paulo: Ed. Rocca; 2010. p. 3-30.

3. Shapero BG, Black SK, Liu RT, Klugman J, Bender RE, Abramson LY, Alloy LB. Stressful life events and depression symptoms: the effect of childhood emotional abuse on stress reactivity. J Clin Psychol 2014; 70(3):209-223.

4. Jansen K, Cardoso Tde A, Mondin TC, Matos MB, Souza LD, Pinheiro RT, Magalhães PV, Silva RA. Stressful life events and mood disorders: a community sample. Cien Saude Colet 2014; 19(9):3941-3946.

5. Sparrenberger F, Santos I, Lima RC. Associação de eventos de vida produtores de estresse e mal-estar psicológico: um estudo de base populacional. Cad Saude Publica 2004; 20(1):249-258.

6. Rosso G, Albert U, Asinari GF, Bogetto F, Maina G. Stressful life events and obsessive-compulsive disorder: clinical features and symptom dimensions. Psychiatry Res 2012; 197(3):259-264

7. Moitra E, Dyck I, Beard C, Bjornsson AS, Sibrava NJ, Weisberg RB, Keller MB. Impact of stressful life events on the course of panic disorder in adults. J Affect Disord 2011; 134(1-3):373-376.

8. Fortes S, Villano LAB, Lopes CS. Nosological profile and prevalence of common mental disorders of patients seen at the Family Health Program (FHP) units in Petrópolis, Rio de Janeiro. Rev Bras Psiquiatr 2008; 30(1):32-37.

9. Fortes S, Lopes CS, Villano LAB, Campos MR, Gonçalves DA, Mari JJ. Common mental disorders in Petrópolis-RJ: a challenge to integrate mental health into primary care strategies. Rev Bras Psiquiatr 2011; 33(2):150-156.

10. Lopes CS, Faerstein E, Chor D. Eventos de vida produtores de estresse e transtornos mentais comuns: resultados do Estudo Pró-Saúde. Cad Saude Publica 2003; 19(6):1713-1720.

11. Lima MS, Beria JU, Tomasi E, Conceicao AT, Mari JJ. Stressful life events and minor psychiatric disorders: an estimate of the population attributable fraction in a Brazilian community-based study. Int J Psychiatry Med 1996; 26(2):211-222.

12. Corless IB, Voss J, Guarino AJ, Wantland D, Holzemer W, Jane Hamilton M, Sefcik E, Willard S, Kirksey K, Portillo C, Rivero Mendez M, Rosa ME, Nicholas PK, Human S, Maryland M, Moezzi S, Robinson L, Cuca Y. The Impact of Stressful Life Events, Symptom Status, and Adherence Concerns on Quality of Life in People Living With HIV. J Assoc Nurses AIDS Care 2013; 24(6):478-490.

13. Johansen VA, Wahl AK, Eilertsen DE, Weisaeth L, Hanestad BR. The predictive value of post-traumatic stress disorder symptoms for quality of life: a longitudinal study of physically injured victims of non-domestic violence. Health Qual Life Outcomes 2007; 5:26.

14. Moreira TdC, Figueiró LR, Fernandes S, Justo FM, Dias IR, Barros HMT, Ferigolo M. Quality of life of users of psychoactive substances, relatives, and non-users assessed using the WHOQOL-BREF. Cien Saude Colet 2013; 18(7):1953-1962.
15. Archea C, Yen IH, Chen H, Eisner MD, Katz PP, Masharani U, Yelin EH, Earnest G, Blanc PD. Negative life events and quality of life in adults with asthma. Thorax 2007; 62(2):139-146.

16. Caron J, Lecomte Y, Stip E, Renaud S. Predictors of quality of life in schizophrenia. Community Ment Health J 2005; 41(4):399-417.

17. Minayo MCS, Hartz ZMA, Buss PM. Qualidade de vida e saúde: um debate necessário. Cien Saude Colet 2000; 5(1):7-18

18. TheWHOQOLGroup. The World Health Organization quality of life assessment (WHOQOL): position paper from the World Health Organization. Soc Sci Med 1995; 41(10):1403-1409.

19. Zhan L. Quality of life: conceptual and measurement issues. J Adv Nurs 1992; 17(7):795-800.

20. Fortes S. Relatório Final: Avaliação de um modelo de capacitação em saúde mental na atenção básica: cuidados integrais na prática do matriciamento. Brasília: Conselho Nacional de Desenvolvimento Científico e Tecnológico; 2009.

21. Goncalves DA, Fortes S, Campos M, Ballester D, Portugal FB, Tofoli LF, Gask L, Mari J, Bower P. Evaluation of a mental health training intervention for multidisciplinary teams in primary care in Brazil: a pre- and posttest study. Gen Hosp Psychiatry 2013; 35(3):304308.

22. Goncalves DA, Mari Jde J, Bower P, Gask L, Dowrick C, Tofoli LF, Campos M, Portugal FB, Ballester D, Fortes S. Brazilian multicentre study of common mental disorders in primary care: rates and related social and demographic factors. Cad Saude Publica 2014; 30(3):623632.

23. Portugal FB, Campos MR, Gonçalves DA, Mari JdJ, Gask L, Bower P, Dowrick C, Fortes S. Psychiatric morbidity and quality of life of primary care attenders in two cities in Brazil. Jornal Brasileiro de Psiquiatria 2014; 63(1):23-32.

24. Fortes S. Transtornos mentais na atenção primária: suas formas de apresentação, perfil nosológico e fatores associados em unidades do Programa de Saúde da Família do município de Petrópolis/Rio de Janeiro/Brasil. Rio de Janeiro: Universidade do Estado do Rio de Janeiro; 2004.

25. Fleck MP, Louzada S, Xavier M, Chachamovich E, Vieira G, Santos L, Pinzon V. Aplicação da versão em português do instrumento abreviado de avaliação da qualidade de vida "WHOQOL-bref". Rev Saude Publica 2000; 34(2):178-183.

26. Goldberg DP, Blackwell B. The Detection of Psychiatric Illness by Questionnaire. London: Oxford University Press; 1972.

27. Mari JJ, Williams P. A comparison of the validity of two psychiatric screening questionnaires (GHQ-12 and SRQ-20) in Brazil, using Relative Operating Characteristic (ROC) analysis. Psychol Med 1985; 15(3):651-659.

28. Goldberg D, Huxley P. Common Mental Disorders: a Bio-Social Model. London: Tavistock, Routledge; 1992.

29. Goldberg DP, Blackwell B. Psychiatric illness in general practice. A detailed study using a new method of case identification. Br Med J 1970; 1(5707):439-443. 
30. Botega NJ, Bio MR, Zomignani MA, Garcia Júnior C, Pereira WAB. Transtornos do humor em enfermaria de clínica médica e validação de escala de medida (HAD) de ansiedade e depressão. Rev Saude Publica 1995; 29(5):359-363.

31. Chor D, Griep RH, Lopes CS, Faerstein E. Medidas de rede e apoio social no Estudo Pró-Saúde: pré-testes e estudo piloto. Cad Saude Publica 2001; 17(4):887-896.

32. Azevedo ALS, Silva RA, Tomasi E, Quevedo LA. Doenças crônicas e qualidade de vida na atenção primária à saúde. Cad Saude Publica 2013; 29(9):1774-1782.

33. Berlim MT, McGirr A, Fleck MP. Can sociodemographic and clinical variables predict the quality of life of outpatients with major depression? Psychiatry Res 2008; 160(3):364-371.

34. Galvão LLLF, Farias MCS, Azevedo PRM, Vilar MJP, Azevedo GD. Prevalência de transtornos mentais comuns e avaliação da qualidade de vida no climatério. Rev Associação Médica Brasileira 2007; 53(5):414-420.

35. Laguardia J, Campos MR, Travassos CM, Najar AL, Anjos LA, Vasconcellos MM. Psychometric evaluation of the SF-36 (v.2) questionnaire in a probability sample of Brazilian households: results of the survey Pesquisa Dimensoes Sociais das Desigualdades (PDSD), Brazil, 2008. Health Qual Life Outcomes 2011; 9:61.

36. Valla VV. Educação popular, saúde comunitária e apoio social numa conjuntura de globalização. Cad Saude Publica 1999; 15(Supl.):S7-S14.

37. Costa AGd, Ludermir AB. Transtornos mentais comuns e apoio social: estudo em comunidade rural da Zona da Mata de Pernambuco, Brasil. Cad Saude Publica 2005; 21(1):73-79.

38. Epel OB, Kaplan G, Moran M. Perceived discrimination and health-related quality of life among Arabs and Jews in Israel: a population-based survey. BMC Public Health 2010; 10:282.

39. Gordia AP, Quadros TMB, Campos W. Variáveis sociodemográficas como determinantes do domínio meio ambiente da qualidade de vida de adolescentes. Cien Saude Colet 2009; 14(6):2261-2268.
40. Sorensen J, Kruse M, Gudex C, Helweg-Larsen K, Bronnum-Hansen $\mathrm{H}$. Physical violence and health-related quality of life: Danish cross-sectional analyses. Health Qual Life Outcomes 2012; 10(1):113.

41. D'Souza MS, Karkada SN, Somayaji G. Factors associated with health-related quality of life among Indian women in mining and agriculture. Health Qual Life Outcomes 2013; 11:9.

42. Carvalho A, Buss P. Determinantes Sociais na Saúde, na doença e na intervenção. In: Giovanella L, Escorel S, Lobato L, Noronha J, Carvalho A, editors. Politicas e sistema de saúde no Brasil. Rio de Janeiro: Editora Fiocruz; 2008. p. 141-66.

43. Higginson IJ, Carr AJ. Measuring quality of life: Using quality of life measures in the clinical setting. BMJ 2001; 322(7297):1297-300.

44. Brasil. Ministério da Saúde (MS). Atenção Básica e a Saúde da Família [cited 20139 jan]. Available from: http://dab.saude.gov.br/atencaobasica.php\#saudedafamilia.

45. Brasil. Ministério do Desenvolvimento Social e Combate à Fome (MDS). Sistema Único de Assistência Social (Suas) [cited 201321 jun]. Available from: http:// www.mds.gov.br/assistenciasocial/suas.

Artigo apresentado em 10/04/2014

Aprovado em 15/05/2015

Versão final apresentada em 17/05/2015 